\title{
Visualization of the wake behind a sliding bubble
}

\author{
R. O’Reilly Meehan, ${ }^{1}$ K. Grennan, ${ }^{2}$ I. Davis, ${ }^{1}$ K. Nolan, ${ }^{1}$ and D. B. Murray ${ }^{2}$ \\ ${ }^{1}$ Thermal Management Research Group, Efficient Energy Transfer ( $\eta$ ET) Department, Nokia Bell Labs, \\ Blanchardstown Business \& Technology Park, Snugborough Rd, Dublin 15, Ireland \\ ${ }^{2}$ Department of Mechanical and Manufacturing Engineering, Trinity College Dublin, Dublin 2, Ireland
}

(Received 24 March 2017; published 13 October 2017)

\begin{abstract}
In this work, Schlieren measurements are presented for the wake of an air bubble sliding under a heated, inclined surface in quiescent water to provide new insights into the intricate sliding bubble wake structure and the associated convective cooling process. This is a two-phase flow configuration that is pertinent to thermal management solutions, where the fundamental flow physics have yet to be fully described. In this work, we present an experimental apparatus that enables high-quality Schlieren images for different bubble sizes and measurement planes. By combining these visualizations with an advanced bubble tracking technique, we can simultaneously quantify the symbiotic relationship that exists between the sliding bubble dynamics and its associated wake. An unstable, dynamic wake structure is revealed, consisting of multiple hairpin-shaped vortex structures interacting within the macroscopic area affected by the bubble. As vorticity is generated in the near wake, the bubble shape is observed to recoil and rebound. This also occurs normal to the surface and is particularly noticeable for larger bubble sizes, with a periodic ejection of material from the near wake corresponding to significant shape changes. These findings, along with their implications from a thermal management perspective, provide information on the rich dynamics of this natural flow that cannot be obtained using alternate experimental techniques.
\end{abstract}

DOI: 10.1103/PhysRevFluids.2.104303

\section{INTRODUCTION}

Observation of the dynamics of a gas bubble moving through a liquid provides insight into a complex natural phenomenon that is important to a diverse range of applications in chemical engineering, water treatment, and thermal management [1-4]. Regarding thermal management, it is well established that two-phase flows can offer significant performance advantages, including extremely high heat transfer coefficients, temperature homogenization, and passive and low-power consumptions [5]. However, the coupling of the motion of the gas and liquid phases can lead to difficulty in predicting the behavior of two-phase thermal management solutions, meaning the reliability of such systems cannot be easily ascertained [5]. An understanding of the bubble wake is crucial from a thermal management perspective, since single-phase convective transport has been observed to be at least as important as latent heat in pool boiling [6], while it has been observed to dominate over phase change in more confined geometries [7]. Although the numerical modeling of these flows has proved fruitful [8], the bubble motion in the aforementioned engineering applications is highly sensitive to a wide range of parameters. Thus, there remains a need for high-quality, fundamental experiments that provide insight into the flow behavior and potential modeling strategies.

Bubbles rising in unbound media have undergone significant study in terms of the bubble shape [4,9], path [10,11], dynamics [12,13], wake structures [14-17], and bubble-bubble interactions $[18,19]$. In comparison, those moving in constricted geometries have received less attention. Initial work focused on bubbles moving in vertical or inclined channels, later expanding to bubbles sliding under inclined flat plates [20-22]. The current study focuses on a single air bubble sliding under a heated inclined surface immersed in water. This is an expansion of work on shell and tube heat exchangers, wherein bubbles rise, impact, and subsequently slide along the underside of the 
tubes. Work to date has looked at the various stages of this process for air bubbles impacting flat [23], inclined [24], or vertical [25] heated surfaces. For inclined surfaces, bubbles tend to impact, bounce, and subsequently slide under the surface. To date, both vapor and gas bubbles have been observed to enhance convective heat transfer rates as they slide [6,26-28]. A significant portion of this enhancement stems from the turbulent mixing of the surrounding fluid, which is induced by the unsteady wake [3]. Previous work has sought to capture the dynamics of the sliding bubble and its associated wake by means of infrared (IR) thermography [3,29], bubble interface tracking [1], and particle image velocimetry (PIV) $[1,30]$. To date, a wake structure has been detected that is broadly consistent with the hairpin vortex structure observed behind freely rising bubbles $[15,17,31]$ and near-wall bluff bodies [32,33]. At low surface to bulk temperature differences of $10^{\circ} \mathrm{C}$, convective heat transfer enhancement 7-8 times that of natural convection has been shown to occur in small, discrete "packets" within the wake structure [3,29]. This wake structure has also been found to alter the dynamics of a subsequent trailing sliding bubble, for which the mechanism of heat transfer enhancement is more complex [2].

While the cooling structures observed by IR thermography were broadly consistent with those using PIV, the intricate regions of heat flux enhancement and suppression observed within the wake indicated its dynamics are more complex than observed from two-dimensional (2D) PIV experiments. While 2D PIV provides quantitative in-plane velocity measurements, these are integrated over interrogation windows. Additionally, each window is limited to two dimensions, although it is possible to step the laser position to obtain a pseudo-three-dimensional (3D) representation of the flow field [1]. Thin-foil thermography provides higher resolution measurements but only at the heated surface. Schlieren photography has distinct advantages over these two techniques in that it provides a pseudo-3D view of the fluid motion in the bubble wake and is nonintrusive in the sense that it does not alter the surface tension of the fluid or the slip condition at the bubble surface in the manner that dye and tracer particles do. The technique, which has existed for 150 years [34], has been used to visualize the wakes behind freely rising gas bubbles [31], the fluid motion during the collision between a freely rising bubble and a vertical wall [35] and the wake structures of rising and falling spheres [36].

To date, however, Schlieren measurements have not been performed on a bubble sliding under a heated surface. In addition to providing new insights into the intricate sliding bubble wake structure and the convective cooling process, the thermal gradient between the surface and the bulk liquid enables spatially and temporally resolved Schlieren visualizations. In this work, we present a novel experimental apparatus that enables this measurement, providing sharp Schlieren images for different bubble sizes and measurement planes. By combining these powerful visualizations with an advanced bubble tracking technique, we can simultaneously quantify the coupling between the sliding bubble shape and its associated wake and therefore understand the implications of this symbiotic relationship from a thermal perspective.

\section{EXPERIMENT}

Schlieren imaging is based on the principle that density gradients deviate the path of light rays passing in the vicinity or through these gradients. If these deflected rays are focused onto a suitable filter blocking undeflected light (e.g., a razor blade or knife edge), this gives rise to variations in the distribution of light at the image plane dependent on the deflection of the light beams.

There are two main challenges involved in obtaining Schlieren images of a sliding bubble parallel to the surface. First, the Schlieren system, which comprises a pair of mirrors, high-speed camera, LED light source, and a razor blade [34], must remain normal to the sliding surface, which is to be inclined at $30^{\circ}$ to enable comparison to our previous studies [1-3]. A custom experimental structure is therefore constructed with all of these components mounted onto an aluminium frame with an adjustable angle. Between the two mirrors, a transparent acrylic box with dimensions of $360 \times 180 \times 100 \mathrm{~mm}^{3}$ is bolted onto the inclined frame and filled with distilled water. A schematic showing the top and side views of this apparatus is provided in Fig. 1. The setup in Fig. 1 describes the 


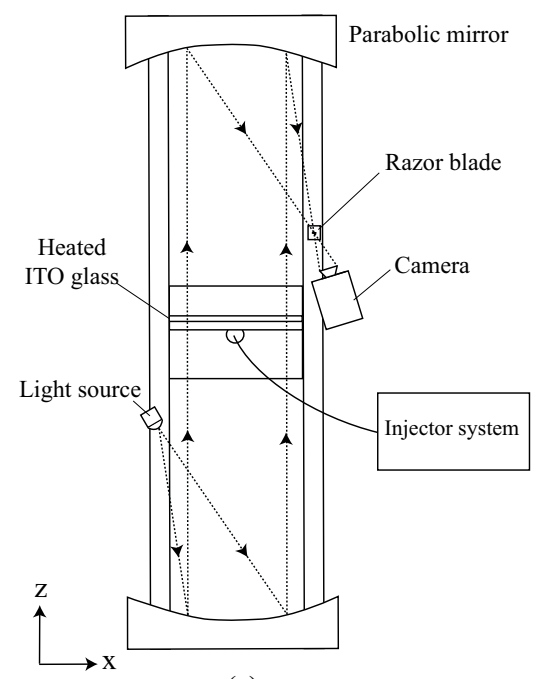

(a)

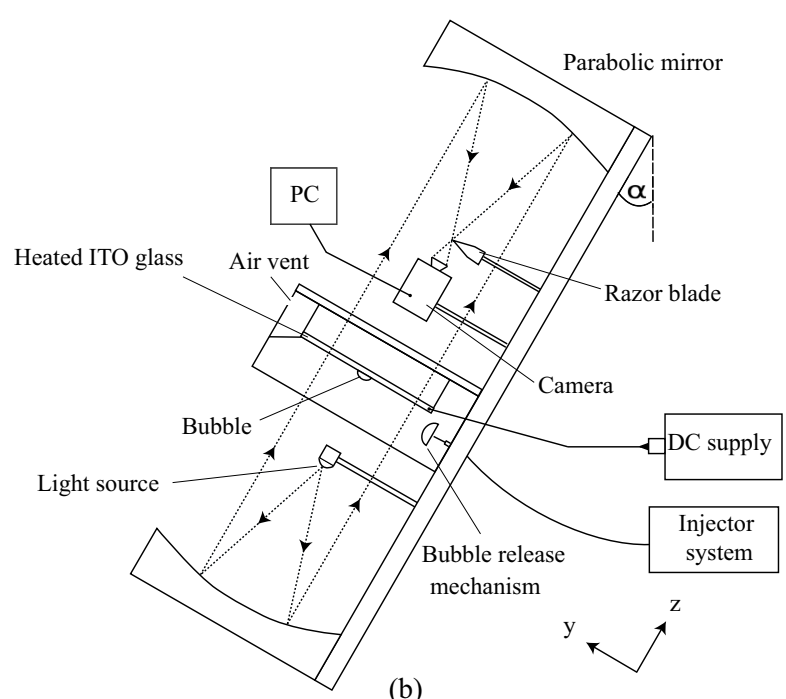

(b)

FIG. 1. Schematic of inclined Schlieren experiment for (a) top and (b) side views, including the $x-y-z$ notation adopted. The surface inclination angle $\alpha$ is $30^{\circ}$ to the horizontal.

wake visualization parallel to the surface. We also perform side view measurements to visualize the motion of the wake structures normal to the surface, wherein the Schlieren apparatus is maintained horizontal and the acrylic tank is inclined at $30^{\circ}$ to the horizontal [note the tank is also rotated by $90^{\circ}$ with respect to Fig. 1(a)].

The second challenge facing implementation of Schlieren for a sliding bubble is the temperature gradient that is required for Schlieren visualization. Previous studies on rising bubbles maintained an artificial temperature gradient across the fluid, with the bubble dragging cool fluid in its wake as it rose [31]. The induced buoyancy force and surface tension variation along the bubble surface imposed by this gradient were shown to be negligibly small [35]. For the case of a sliding bubble, it is advantageous to use a heated surface for this purpose, as the consequent density gradients would be a faithful representation of the actual convective cooling behavior. This is achieved by using a 1.1 mm thick optically transparent indium tin oxide (ITO) coated glass sheet. Passing $35 \mathrm{~W}$ of power across the glass elevates the surface temperature to an average of $8{ }^{\circ} \mathrm{C}$ above the water temperature of $22^{\circ} \mathrm{C}$ through Joule heating, although the surface temperature will not be uniform due to the free convection thermal boundary layer on the underside of the inclined surface.

Bubbles are released to the surface using a 3D printed inverted cup that catches a series of air bubbles injected into the chamber via a system comprising a syringe needle, gastight syringe (model Hamilton 1000 series Gastight, $5 \mathrm{ml}$ ), silicone rubber tubing, and syringe pump (NE-1000 from New Era Pump Systems). A strong magnet is attached to the outside of the cup, meaning it can be rotated from outside the tank by means of a second magnet, meaning minimal disruption of the surrounding fluid. Upon exiting from the bubble release mechanism, the bubble rises $15 \mathrm{~mm}$ before impacting the surface and sliding for $280 \mathrm{~mm}$. We observe the bubble in a $150 \times 100 \mathrm{~mm}^{2}$ window in which it is no longer bouncing. High-speed images are acquired at $1 \mathrm{kHz}$ using a Phantom V311 high-speed camera $(1280 \times 800 \mathrm{CMOS}$ sensor, $20 \mu \mathrm{m}$ pixel size). Following recording, the raw images are postprocessed in MATLAB using the interface tracking algorithm outlined in our previous work [1,2]. This method enables not only the bubble shape and path but the dynamics of its interface to be tracked, thereby linking the fluid shed by the bubble into its near wake to changes in its interface. 
TABLE I. Measured and derived quantities for the three bubbles tested here. In this case, average values are taken for $U_{b}, \chi$, and Re, while $q^{*}$ is the ratio of forced convective heat flux to that of natural convection, measured from previous infrared thermography experiments [3].

\begin{tabular}{lccccccc}
\hline \hline$d_{e}[\mathrm{~mm}]$ & $U_{b}[\mathrm{~mm} / \mathrm{s}]$ & $\chi$ & $\operatorname{Re}$ & Eo & Mo & $q^{*}{ }_{\text {max }}$ & $q^{*}{ }_{\text {mean }, 0.5 s}$ \\
\hline 3 & 151.7 & 0.88 & 453.3 & 1.21 & $2.56 \times 10^{11}$ & - & - \\
5.8 & 171.8 & 0.84 & 993 & 4.52 & $2.56 \times 10^{11}$ & 8.1 & 1.2 \\
9 & 204.7 & 0.55 & 1835 & 10.88 & $2.56 \times 10^{11}$ & - & - \\
9.2 & - & - & - & - & - & 12.3 & 1.56 \\
\hline \hline
\end{tabular}

\section{RESULTS AND DISCUSSION}

In this section, we present Schlieren images of bubbles at equivalent spherical diameters of 3 , 5.8, and $9 \mathrm{~mm}$ sliding under a heated surface inclined at $30^{\circ}$ to the horizontal, which allows for direct comparison to our previous IR and PIV work [1,3,29]. The regime map of Tsao and Koch [37] broadly classifies rising air bubbles of these diameters in water as oblate ellipsoids, although the presence of the sliding surface dramatically alters the bubble shape and behavior. The bubble shape and motion are governed by three important dimensionless groups: the Reynolds, Eötvös, and Morton numbers. These are provided in Table I for each test. Images are provided in a plane parallel to the surface $(x-y)$ and one to the side $(y-z)$ views. Figure 2 shows Schlieren images of bubbles of equivalent diameters $d_{e}=3,5.8$, and $9 \mathrm{~mm}$ respectively in the $x-y$ plane, in each case providing instantaneous snapshots of the fluid motion at three instants in time $0.225 \mathrm{~s}$ apart. Please see videos S1 and S2 in the Supplemental Material [38] for a more complete view of the temporal evolution of this flow field.

Figure 2 illustrates the rich dynamics of the fluid motion within the bubble wake and the complexity of these "looped" structures. In all cases, the bubble takes an undulating path with an unsteady, dynamic wake that overshoots the bubble path line in both the spanwise $(x)$ and length (y) directions. Within this macroscopic wake structure, we observe a series of vortex loops: these will be discussed in greater detail in relation to Fig. 3. For now, we will compare the structures of the three bubbles tested. For the smallest bubble diameter tested $(3 \mathrm{~mm})$, the loops do not appear downstream of the bubble until several bubble diameters, with fluid in the near wake moving in close association with the bubble. Progressing to the $5.8 \mathrm{~mm}$ bubble, we observe the production of more vortex loops, with the transition from the near wake to these hairpin loops occurring far sooner. Finally, the wake structures of the $9 \mathrm{~mm}$ bubble are highly chaotic and turbulent, in which it is more difficult to ascertain individual fluid loops. The unstable behavior of these bubbles indicates that even during what would be considered a nominally steady sliding process, the dynamics of sliding bubbles are inherently unsteady.

Figure 3 explores the temporal evolution of fluid in the near wake of the $5.8 \mathrm{~mm}$ bubble test by mapping the circumferential, mean-subtracted bubble edge velocity magnitude, $v_{\mathrm{mag}}-\overline{v_{\mathrm{mag}}}$, onto the bubble position at 12 instants in time $40 \mathrm{~ms}$ apart. In each image, the coordinate system has been shifted such that the bubble centroid is at the position $y / d_{e}=0$. For further comparison, Fig. 4 provides derived quantities based off the centroid of the bubble, namely, the $x$ component of position, the velocity magnitude $v_{\text {mag }}=\sqrt{v_{x}^{2}+v_{y}^{2}}$, the bubble orientation denoted as the angle made between major axis and positive $x$ axis, and the aspect ratio $\chi=b / a$, where $a$ and $b$ are the major and minor axis lengths, respectively. The values of these quantities corresponding to each image in Fig. 3 are also highlighted by the markers. Figure 4(d) shows that this bubble experiences oscillations between spheroidal and oblate, which are often referred to as mode $(2,0)$ oscillations. How oblate the bubble is at any time is proportional to its velocity magnitude, as has been reported in the literature for rising bubbles [12]. The maximum velocities, and hence minimum aspect ratios, occur when the bubble is at each path extremum, where the velocity vector of the bubble is aligned with its buoyancy force. 
VISUALIZATION OF THE WAKE BEHIND A SLIDING BUBBLE
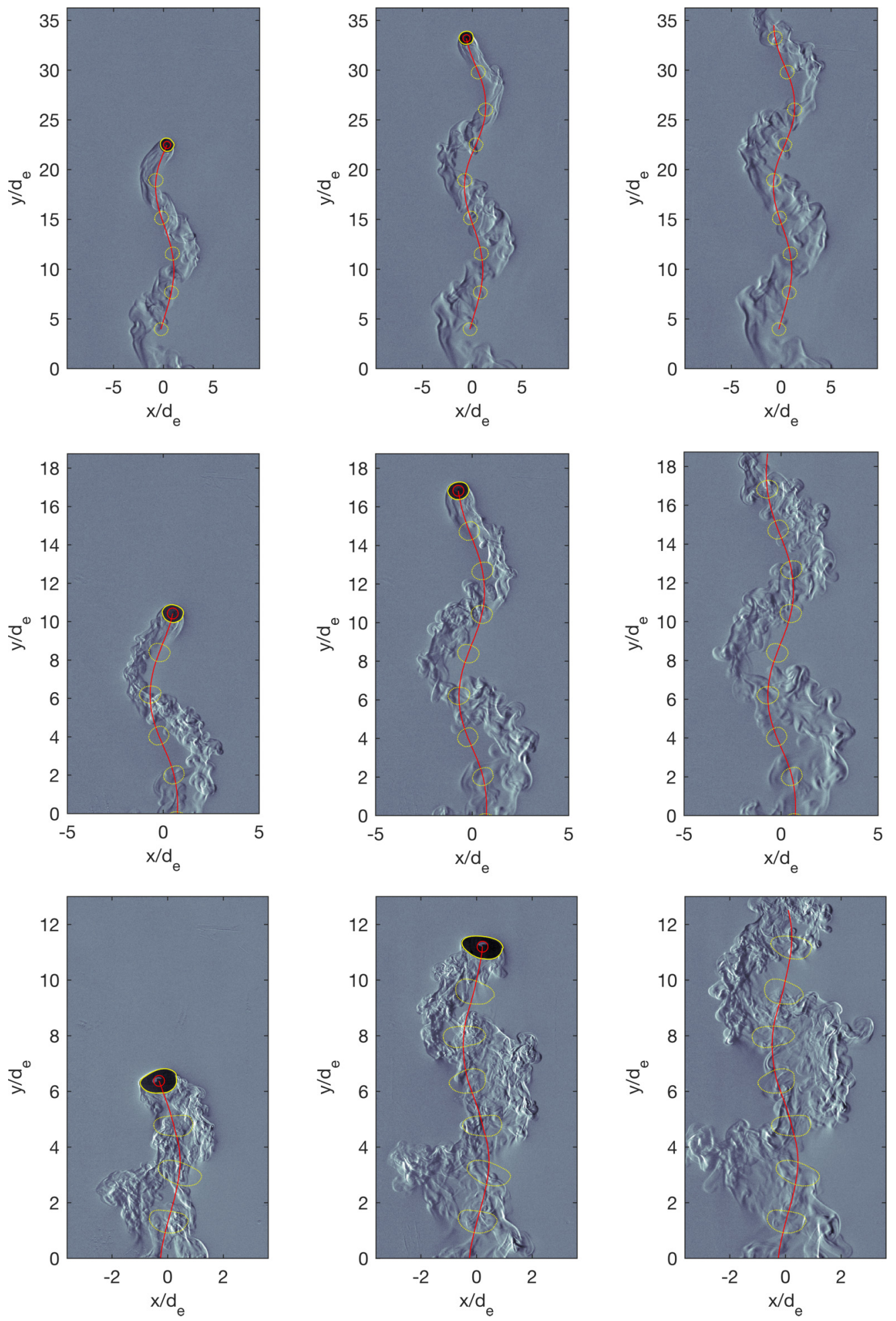

FIG. 2. Surface Schlieren images of sliding bubbles of equivalent diameter $3 \mathrm{~mm}$ (top), $5.8 \mathrm{~mm}$ (middle), and $9 \mathrm{~mm}$ (bottom) taken $0.225 \mathrm{~s}$ apart, along with the bubble path (red line). 


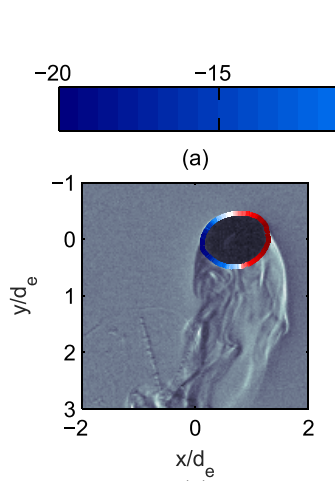

(e)

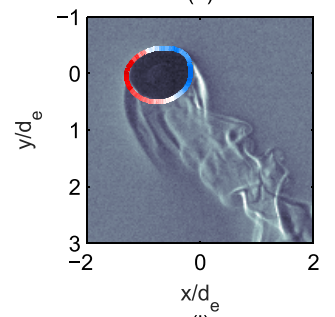

(i)

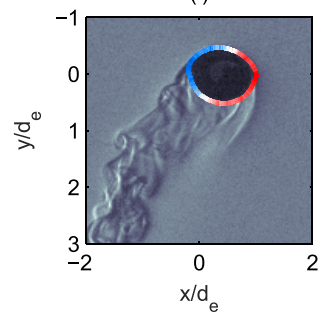

$v_{\text {mag }}-\bar{v}_{\text {mag }}[\mathrm{mm} / \mathrm{s}]$

$-10$

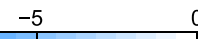

(b)

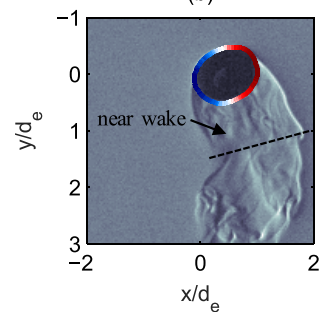

(f)

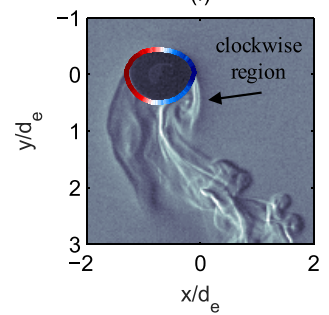

(j)

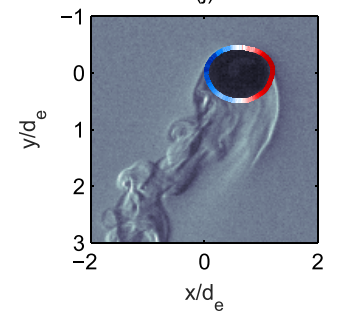

(c)

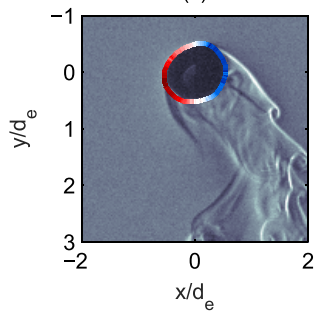

(g)

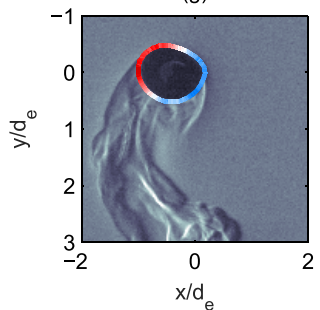

$(\mathrm{k})^{\mathrm{e}}$

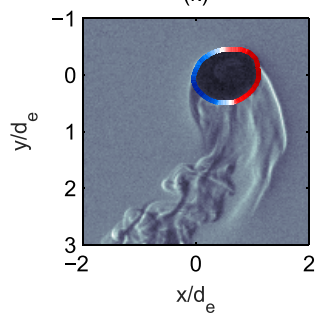

10

(d)

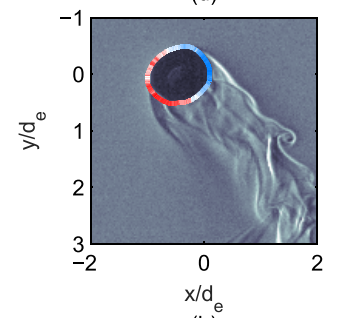

(h)

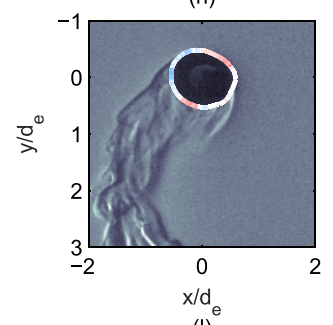

(I)

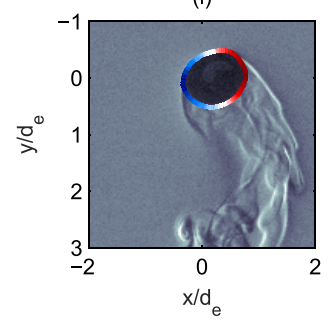

FIG. 3. Surface Schlieren images of the near wake of a $5.8 \mathrm{~mm}$ equivalent diameter sliding bubble taken $40 \mathrm{~ms}$ apart. At each instant in time, the local mean-subtracted bubble edge velocity magnitude, $v_{\text {mag }}-\overline{v_{\text {mag }}}$, is mapped onto the image. For clarity, the bubble centroids are set to the position $y / d_{e}=0$.

As the bubble travels from one extremum to another in Fig. 3, the outside edge of the bubble has a larger velocity since it sweeps out a larger distance. For steadily sliding bubbles of this size, we do not observe significant deformations of the interface, unless the bubble is captured in the wake of another [2]. Figure 3(b) highlights the distinction we make between the near wake and remainder of the wake structure, although this point is not always well defined. At the path inversions, fluid separates from the near wake, with vortex loops forming within this region and being advected

(a)

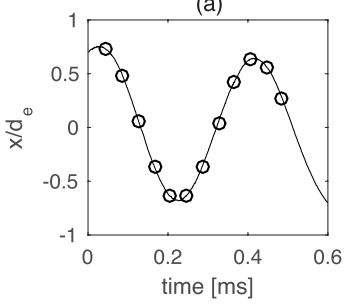

(b)

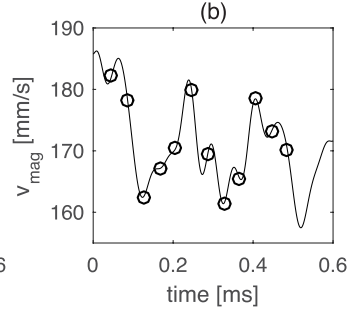

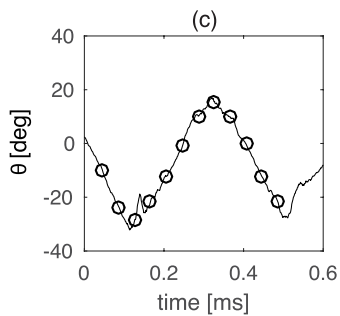

(d)

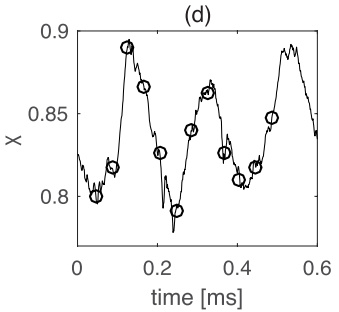

FIG. 4. Derived quantities for a $5.8 \mathrm{~mm}$ bubble: (a) $x$ component of position, (b) velocity magnitude, (c) bubble orientation, and (d) aspect ratio $\chi=b / a$. The markers on each plot refer to each of the images in Fig. 3 . 


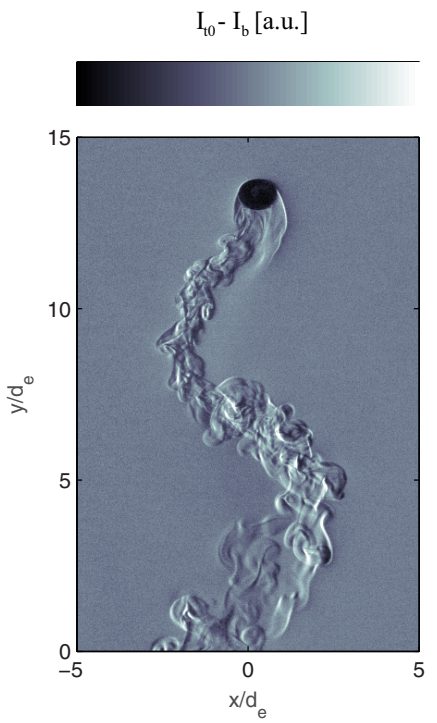

(a)

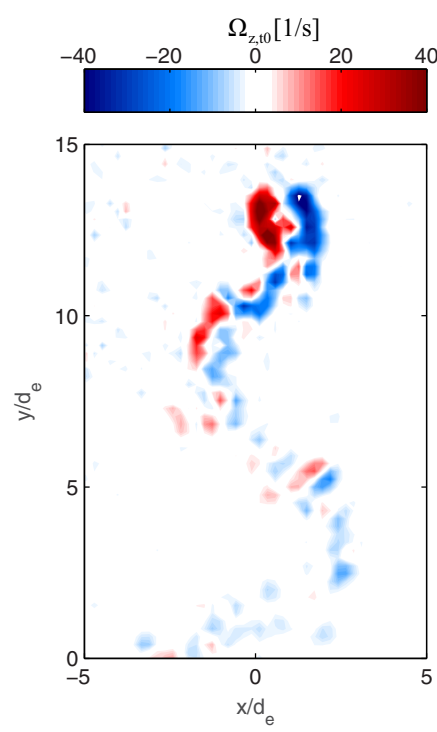

(b)

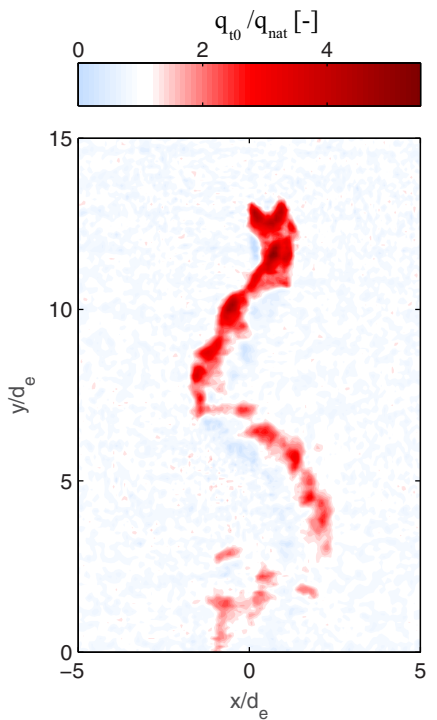

(c)

FIG. 5. Comparison of the wake structures of $5.8 \mathrm{~mm}$ sliding bubbles using different experimental techniques. (a) Schlieren photography from the current study, (b) vorticity, $\Omega_{z, t 0}=$ curl(U) calculated using PIV [1], (c) heat flux enhancement relative to free convection, $q_{t 0} / q_{\text {nat }}$ taken directly from previous thin-foil IR thermography measurements [29].

towards the outside of the path [Figs. 3(c) and 3(d)]. This fluid overshoots the bubble path due to both the momentum of the bubble and the thermal boundary layer that exists at the surface. Within the wake structure, this results in a smoother wake boundary on the inside of the bubble path and the hairpin loops being advected on the outside of the wake. As the bubble changes direction in Figs. 3(f) and $3(\mathrm{~g})$, we observe a clockwise rotating region of fluid developing on the right-hand side of the bubble. This wake behavior corresponds to the bubble tilting in a clockwise direction [Fig. 4(c)], and leads to a further deceleration of the bubble, since its velocity vector becomes misaligned with its buoyancy force. Throughout Fig. 3, the generation of vorticity in the wake is not uniform due to its inherent instability and turbulence. Due to the symbiotic relationship between the bubble and its wake, this means that the dynamics of such bubbles do not exhibit regular temporal fluctuations about some mean value, and instead are prone to large fluctuations corresponding to the build-up and discharge of fluid into its wake.

In Fig. 5 we examine the wake structures of $5.8 \mathrm{~mm}$ sliding bubbles using Schlieren (a), PIV (b), and IR thermography (c). In each case, the surface is inclined at $30^{\circ}$ to the horizontal, and the bubble is at a time $t_{0}$ where it is just past its local maximum in spanwise position. Figure $5(\mathrm{~b})$ presents the vorticity, $\Omega_{z}=\operatorname{curl}(\mathbf{U})$, in the wake of a bubble sliding under an unheated surface. The raw PIV images are taken from our previous work [1], which also contains a full description of the experimental procedure. The vectors are evaluated using the Insight $4 G V 3 V$ PIV package provided by TSI using a window that deforms from $32 \times 32$ to $16 \times 16$ pixels. This small window size is used to visualize the vortical structures within the wake in greater detail, while our previous work used a larger window size with a focus on maintaining temporal coherence and no recursive hole filling [1].

For rising bubbles, numerical studies observed chains of multiple hairpin loops being shed per period of oscillation [17], which are consistent with the inclined Schlieren results provided in Figs. 2, 3 , and 5(a) and with previous Schlieren work on rising bubbles [31]. However, prior PIV approaches for rising [15] or sliding [1] bubbles have observed only a single structure separating from the 
near wake at the bubble path inversions. These differences were initially attributed by Gaudlitz and Adams [17] to surfactants, which alter the surface tension of the water and impose a partial slip boundary condition at the bubble interface. However, we postulate that it is the low pass filtering inherent to 2D PIV that result in a different wake structure being ascertained, not the influence of surfactants as previously reported. This is due to the inherent limitations of planar PIV: namely, the loss in resolution associated with discretizing of the wake into interrogation windows and the fact that only a 2D plane of the wake is being analyzed. Even when the resolution is increased, the vorticity component of these loops may be small and cannot be detected. As such, while 2D PIV tracks the fluid motion of the primary wake structures accurately on a macroscopic level, it does not resolve the secondary 3D hairpin loops formed within the wake due to difficulties in resolution and 2D nature of the measurement. While it is true that Tomographic PIV could provide 3D information on the flow, its implementation would likely prove extremely challenging in this case, since the bubble would block much of the laser light.

Figure 5(c) presents the convective heat flux enhancement relative to free convection from our previous thin-foil IR thermography measurements [29] for the same bubble size. These findings are in excellent agreement with our Schlieren measurements, revealing complex, elliptical-shaped "packets" of cooling $[3,29]$ within the wake structure itself. Small regions of maximum convective heat flux enhancement are found to occur on the outside of the bubble path, which is consistent with the advection and motion of the hairpin loops within the wake structure observed using Schlieren measurements. In addition, the shapes of the cooling structures are in agreement with the impact of the hairpin vortex heads on the surface observed using Schlieren.

Along with the derived quantities previously discussed in Fig. 3, Table I also presents more quantitative measurements of convective heat transfer enhancement to precipitate future research into the optimization of sliding bubble based cooling applications. These have been obtained from previous IR thermography measurements and are presented as the ratio of forced to free convection, or $q^{*}$. The average value in this case refers to a $20 \mathrm{~mm} \times 20 \mathrm{~mm}$ region through which the bubbles pass. We observe larger peak and average values for the larger bubble size, although the maximum values are transient due to the rapidly fluctuating convective heat flux, which was also observed numerically by Kim et al. [24] using the VOF method. While little modeling work exists on the flow structures of large sliding bubbles, the complexity and significant motion of the flow structures observed herein are in agreement with the modeling of Zun et al. [14], who studied the interaction of the wake of a $6 \mathrm{~mm}$ freely rising bubble and a vertical heated wall. The authors observed a chain of interconnected vortex rings with a significantly larger amplitude than that of the bubble path. The wake impact on the wall was found to be time-dependent, as the legs of the quasiperiodic shedding varied by intensity when measured on a fixed plane, which was also measured in our previous IR experiments and is indicated by the unsteady flow structures observed in the current study. The authors also observed strong lateral mixing, with a significant time scale due to the long lifetime of the bubble wake. The authors concluded that the bulk liquid thermal agitation could not be correctly modelled without first considering the bubble-size-dependent 3D wake structure.

Figure 6 presents side view Schlieren images for equivalent diameters of $5.8 \mathrm{~mm}$ and $9 \mathrm{~mm}$ to show the motion of fluid normal to the surface. Note that these images have been rotated $30^{\circ}$ clockwise for easier comparison and that the bubble path oscillations are confined to the $x-y$ plane and are not apparent from this viewpoint. For the $5.8 \mathrm{~mm}$ bubble, we observe that the motion of the wake normal to the surface commences only at $\sim 2$ diameters downstream of the bubble (i.e., outside of the near wake defined in Fig. 2), while the initial fluid motion remains close to the surface. Additionally, significant fluid motion in the normal direction is observed at discrete locations corresponding to the bubble path extrema. Both of these findings are consistent with previous PIV measurements that looked at the motion in an $x-z$ plane [1]. We observe that the bubble shape takes a low height of $3 \mathrm{~mm}$ from the surface and experiences some minor shape fluctuations normal to the surface. This is in agreement with the Volume of Fluid-Continuum Surface Force (VOF-CSF) measurements performed by Senthilkumar et al. [39], albeit for a smaller bubble size of $3.4 \mathrm{~mm}$. 
(i)

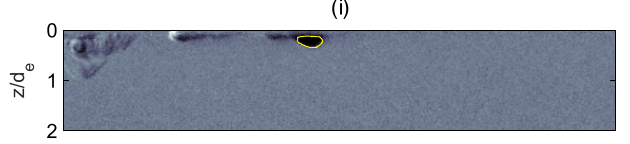

(ii)

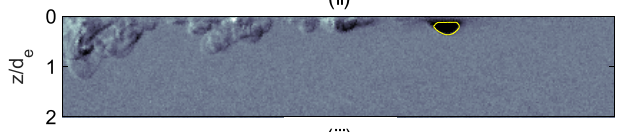

(iii)

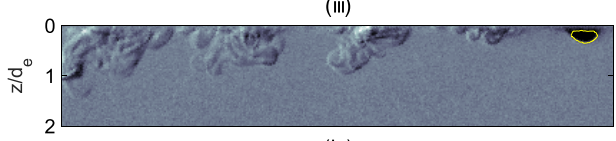

(iv)

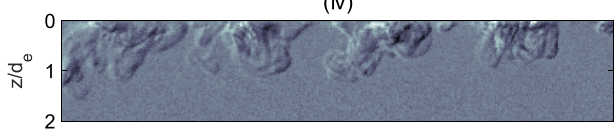

(v)

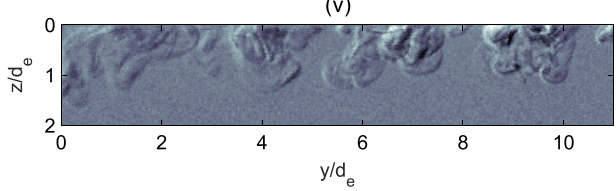

(a)

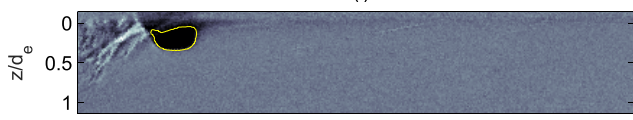

(ii)

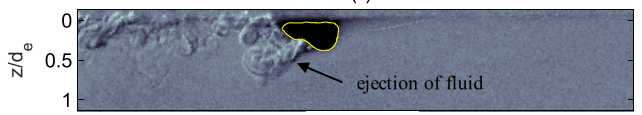

(iii)

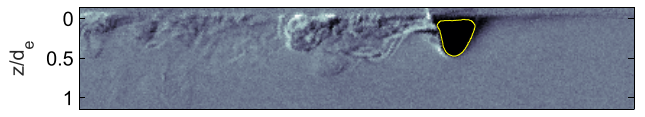

(iv)

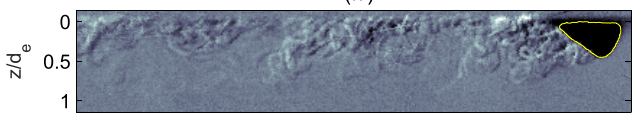

(v)

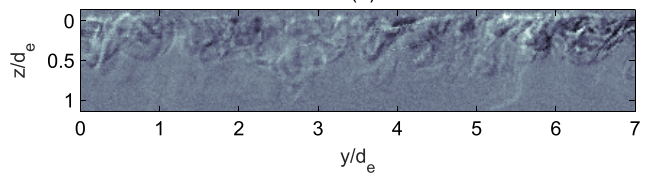

(b)

FIG. 6. Side view Schlieren images for a sliding bubble of equivalent diameter $d_{e}=$ (a) 5.8 and (b) $9 \mathrm{~mm}$ taken $75 \mathrm{~ms}$ apart. The images are rotated $30^{\circ}$ clockwise, while the heated surface is at the position $z / d_{e}=0$.

For the $9 \mathrm{~mm}$ bubble, however, a significant contrast is observed. The bubble undergoes significant shape oscillations, with a maximum height of nearly $5 \mathrm{~mm}$ and open, turbulent wake structure.

Figure 7 focuses on the near wake of the $9 \mathrm{~mm}$ bubble at 12 instances in time $5 \mathrm{~ms}$ apart, starting $50 \mathrm{~ms}$ before Fig. 6(b) (ii), along with the bubble boundary at its maximum height, $h / d_{e}$. We observe the bubble shape growing in the $z$ direction until it reaches its maximum height in Fig. 7(d), where the bubble shape approaches that of an inverted equilateral triangle. During this process, fluid advection is observed in the near wake in a region that is approximately half the height of the bubble. During the next 20 ms [Figs. 7(e)-7(h)], we observe the steady generation of a clockwise vortex loop to the rear of the bubble at its position of maximum height. At this same location, the bubble interface experiences a deformation in the opposite direction, resulting in a bubble shape that is asymmetric about its $y$ axis. The bubble interface responds to this large deformation by recoiling, as highlighted on Figs. 7(i) and 7(j). Finally, the attached vortex separates from the near wake of the bubble, with its clockwise direction of motion acting to drag cool fluid from the bulk and introduce it to the surface [Figs. 7(k) and 7(1)]. At this instant, the bubble shape flattens to its lowest height, although the bubble shape remains asymmetric. The plot of $h / d_{e}$ shows that this process is periodic. The extent of bubble motion normal to the surface is key to the turbulent mixing offered by large sliding bubbles. Furthermore, the aforementioned symbiotic relationship between the bubble and its wake is shown to be very much a 3D process. Please see video S3 of the Supplemental Material [38] for further information.

To summarize, we observe an overall wake structure for a sliding bubble that is consistent with previous PIV measurements [1], with fluid ejected from the near wake at its path extrema and subsequently overshooting the path. However, our Schlieren measurements also reveal the generation of multiple hairpin loops within the wake per path wavelength cycle. This agrees with previous Schlieren experiments [31] and numerical studies [17] for rising bubbles, but are not observed by studies using 2D PIV [1,15]. We postulate that rather than the influence of surfactants affecting results, it is that 2D PIV is limited in its ability to track these hairpin loops due to its inherent $2 \mathrm{D}$ nature and low-pass filtering. The impact of these loops on this surface describes the 
(a)

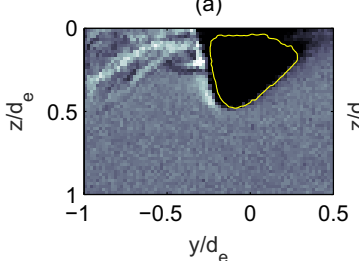

(e)

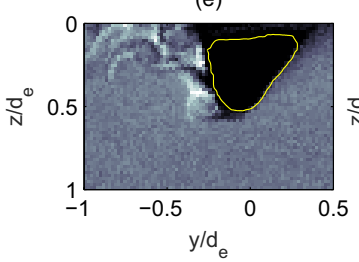

(i)

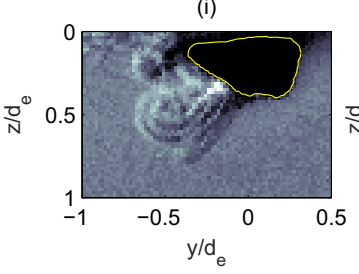

(b)

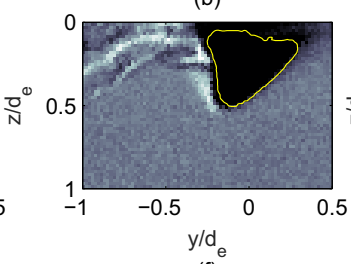

(f)

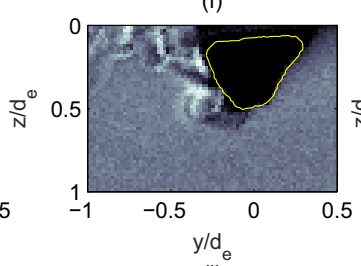

(j)

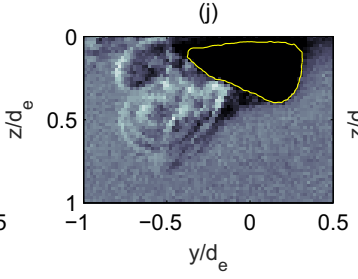

(c)

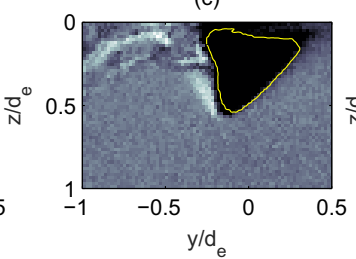

(g)

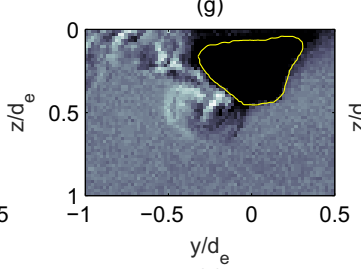

(k)

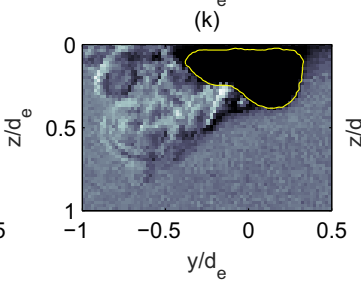

(d)

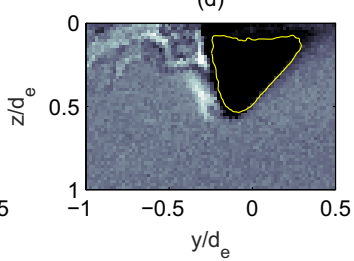

(h)

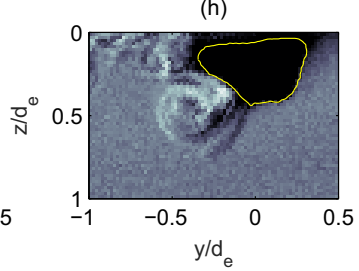

(I)

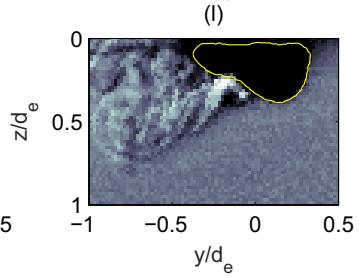

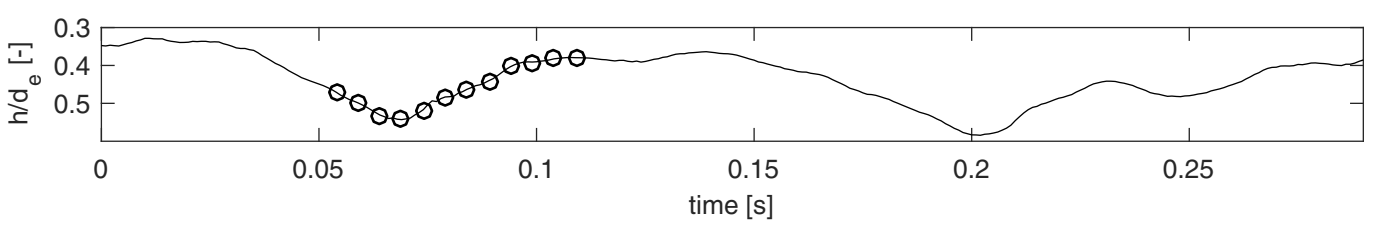

FIG. 7. Near wake of the $9 \mathrm{~mm}$ bubble, with each image $5 \mathrm{~ms}$ apart. The bubble centroid in each case is at the position $y / d_{e}=0$. Also provided is the height of the bubble below the surface, $h / d_{e}$, with the height at each of these 12 instances highlighted.

discrete regions of cooling observed at the surface in previous IR experiments [3,29]. The generation of vorticity to the rear of the bubble has a profound effect on the dynamics of its interface, which is observed to recoil and rebound as it traverses the surface. We observe that the dynamics of sliding bubbles are not steady, and instead involve a 3D process that depends on the complex build-up and shedding of fluid in the bubble wake. Finally, we observe significant fluid disruption and the motion of hairpin vortex loops normal to the surface, which is consistent with measurements of bluff bodies in the literature [32,33]. We believe that this work has highlighted the significant advantages of Schlieren as a technique in studying two-phase systems, and in future work seek to improve this technique by employing methods to extract flow quantities (e.g., wake velocity) from Schlieren data.

\section{CONCLUSION}

This work demonstrates Schlieren measurements of single sliding bubbles for different bubble sizes and with two measurement planes. An experimental setup is used to obtain high-quality Schlieren images that show the dynamics of this two-phase system without the need for an artificial temperature gradient. These images are combined with an advanced bubble tracking technique to simultaneously quantify the coupling between the sliding bubble shape and its associated wake for the first time. We reveal an unstable, dynamic wake structure with no obvious vortex shedding frequency. Downstream of the bubble, the wake takes the form of a series of hairpin loops that are advected laterally and normal to the surface. Multiple hairpin vortices are observed within 
each wavelength cycle. As vorticity is generated in the near wake, the bubble shape is observed to recoil and rebound, indicating that the dynamics of sliding bubbles are not steady. These Schlieren measurements highlight the 3D coupling that exists between the bubble, its wake, and the surface, and provide insights into the rich dynamics of this natural flow that cannot be readily observed using alternate flow visualization or measurement techniques.

\section{ACKNOWLEDGMENTS}

Nokia Bell Labs would like to thank IDA Ireland for their financial support.

[1] R. O'Reilly Meehan, B. Donnelly, K. Nolan, T. Persoons, and D. B. Murray, Flow structures and dynamics in the wakes of sliding bubbles, Int. J. Multiphase Flow 84, 145 (2016).

[2] R. O'Reilly Meehan, B. Donnelly, K. Nolan, and D. B. Murray, Bubble-wake interactions of a sliding bubble pair and the mechanisms of heat transfer, Int. J. Heat Mass Transf. 108, 1347 (2017).

[3] B. Donnelly, R. O'Reilly Meehan, K. Nolan, and D. B. Murray, The dynamics of sliding air bubbles and the effects on surface heat transfer, Int. J. Heat Mass Transf. 91, 532 (2015).

[4] L. Fan and K. Tsuchiya, Bubble Wake Dynamics in Liquids and Liquid-Solid Suspensions (ButterworthHeinemann, Stoneham, 1990).

[5] A. J. Robinson, A thermal-hydraulic comparison of liquid microchannel and impinging liquid jet array heat sinks for high-power electronics cooling, IEEE Tran. Components Packaging Tech. 32, 347 (2009).

[6] S. Houston and K. Cornwell, Heat transfer to sliding bubbles on a tube under evaporating and nonevaporating conditions, Int. J. Heat Mass Transf. 39, 211 (1996).

[7] K. E. Albahloul, D. K. Hollingsworth, L. C. Witte, and A. B. Ozer, Comparing the enhancement of heat transfer caused by sliding gas bubbles and by sliding vapor bubbles in subcooled flow in a minichannel, in ASME 2013 Heat Transfer Summer Conference (American Society of Mechanical Engineers, Minneapolis, $\mathrm{MN}, 2013)$.

[8] D. Bothe, M. Koebe, K. Wielage, and H.-J. Warnecke, Vof-simulations of mass transfer from single bubbles and bubble chains rising in aqueous solutions, in ASME/JSME 2003 4th Joint Fluids Summer Engineering Conference (American Society of Mechanical Engineers, Honolulu, Hawaii, 2003), pp. 423-429.

[9] D. Bhaga and M. Weber, Bubbles in viscous liquids: Shapes, wakes and velocities, J. Fluid Mech. 105, 61 (1981).

[10] G. Mougin and J. Magnaudet, Path Instability of a Rising Bubble, Phys. Rev. Lett. 88, 014502 (2001).

[11] G. Mougin and J. Magnaudet, Wake-induced forces and torques on a zigzagging/spiralling bubble, J. Fluid Mech. 567, 185 (2006).

[12] R. Clift, J. R. Grace, and M. E. Weber, Bubbles, Drops, and Particles (Courier Corporation, New York, 2005).

[13] A. Tomiyama, G. Celata, S. Hosokawa, and S. Yoshida, Terminal velocity of single bubbles in surface tension force dominant regime, Int. J. Multiphase Flow 28, 1497 (2002).

[14] I. Zun, M. Perpar, J. Gregorc, K. Hayashi, and A. Tomiyama, Mixing of thermally stratified water layer by a free rising wobbling air bubble, Chem. Eng. Sci. 72, 155 (2012).

[15] C. Brücker, Structure and dynamics of the wake of bubbles and its relevance for bubble interaction, Phys. Fluids 11, 1781 (1999).

[16] T. Sanada, M. Shirota, and M. Watanabe, Bubble wake visualization by using photochromic dye, Chem. Eng. Sci. 62, 7264 (2007).

[17] D. Gaudlitz and N. A. Adams, Numerical investigation of rising bubble wake and shape variations, Phys. Fluids 21, 122102 (2009).

[18] C. Stewart, Bubble interaction in low-viscosity liquids, Int. J. Multiphase Flow 21, 1037 (1995). 
[19] K. Tsuchiya, T. Miyahara, and L. Fan, Visualization of bubble-wake interactions for a stream of bubbles in a two-dimensional liquid-solid fluidized bed, Int. J. Multiphase Flow 15, 35 (1989).

[20] T. Maxworthy, Bubble rise under an inclined plate, J. Fluid Mech. 229, 659 (1991).

[21] A. Peron, L. Kiss, and S. Poncsák, An experimental investigation of the motion of single bubbles under a slightly inclined surface, Int. J. Multiphase Flow 32, 606 (2006).

[22] B. Podvin, S. Khoja, F. Moraga, and D. Attinger, Model and experimental visualizations of the interaction of a bubble with an inclined wall, Chem. Eng. Sci. 63, 1914 (2008).

[23] D. Donoghue, A. Albadawi, Y. Delauré, A. Robinson, and D. B. Murray, Bubble impingement and the mechanisms of heat transfer, Int. J. Heat Mass Transf. 71, 439 (2014).

[24] J. Kim and J. S. Lee, Surface-wettability-induced sliding bubble dynamics and its effects on convective heat transfer, Appl. Thermal Eng. 113, 639 (2017).

[25] D. Donoghue, B. Donnelly, and D. B. Murray, The enhancement effects of a plume of rising bubbles on natural convection from a heated vertical plate, J. Enhanced Heat Transf. 19, 379 (2012).

[26] K. Cornwell and I. Grant, Heat transfer to bubbles under a horizontal tube, Int. J. Heat Mass Transf. 41, 1189 (1998).

[27] B. Bayazit, D. Hollingsworth, and L. Witte, Heat transfer enhancement caused by sliding bubbles, J. Heat Transf. 125, 503 (2003).

[28] D. K. Hollingsworth, L. C. Witte, and M. Figueroa, Enhancement of heat transfer behind sliding bubbles, J. Heat Transf. 131, 121005 (2009).

[29] R. O’Reilly Meehan, N. P. Williams, B. Donnelly, T. Persoons, K. Nolan, and D. B. Murray, Forced convection in the wakes of impacting and sliding bubbles, Heat and Mass Transfer (2017), doi: 10.1007/s00231-017-2165-6.

[30] D. Qiu and V. Dhir, Experimental study of flow pattern and heat transfer associated with a bubble sliding on downward facing inclined surfaces, Exp. Thermal Fluid Sci. 26, 605 (2002).

[31] C. Veldhuis, A. Biesheuvel, and L. Van Wijngaarden, Shape oscillations on bubbles rising in clean and in tap water, Phys. Fluids 20, 040705 (2008).

[32] B. Stewart, M. Thompson, T. Leweke, and K. Hourigan, Numerical and experimental studies of the rolling sphere wake, J. Fluid Mech. 643, 137 (2010).

[33] M. Acarlar and C. Smith, A study of hairpin vortices in a laminar boundary, J. Fluid Mech. 175, 1 (1987).

[34] G. S. Settles, Schlieren and Shadowgraph Techniques: Visualizing Phenomena in Transparent Media (Springer Science \& Business Media, Berlin, 2012).

[35] A. De Vries, A. Biesheuvel, and L. Van Wijngaarden, Notes on the path and wake of a gas bubble rising in pure water, Int. J. Multiphase Flow 28, 1823 (2002).

[36] C. Veldhuis and A. Biesheuvel, An experimental study of the regimes of motion of spheres falling or ascending freely in a Newtonian fluid, Int. J. Multiphase Flow 33, 1074 (2007).

[37] H. Tsao and D. Koch, Observations of high Reynolds number bubbles interacting with a rigid wall, Phys. Fluids 9, 44 (1997).

[38] See Supplemental Material at http://link.aps.org/supplemental/10.1103/PhysRevFluids.2.104303 for video sequences corresponding to 3 of these tests.

[39] S. Senthilkumar, Y. M. C. Delaure, D. B. Murray, and B. Donnelly, The effect of the VOF-CSF static contact angle boundary condition on the dynamics of sliding and bouncing ellipsoidal bubbles, Int. J. Heat Fluid Flow 32, 964 (2011). 